\title{
Correction to: Cytoplasmic LXR expression is an independent marker of poor prognosis for patients with early stage primary breast cancer
}

\author{
Wanting Shao ${ }^{1,2} \cdot$ Christina Kuhn $^{3} \cdot$ Doris Mayr $^{4} \cdot$ Nina Ditsch $^{3} \cdot$ Magdalena Kailuwait $^{1} \cdot$ Verena Wolf $^{1}$. \\ Nadia Harbeck ${ }^{1} \cdot$ Sven Mahner ${ }^{1} \cdot$ Udo Jeschke $^{1,3}$ (1) $\cdot$ Vincent Cavaillès $^{5} \cdot$ Sophie Sixou ${ }^{1,6,7}$
}

Published online: 16 July 2021

(c) Springer-Verlag GmbH Germany, part of Springer Nature 2021

\section{Correction to: \\ Journal of Cancer Research and Clinical Oncology https://doi.org/10.1007/s00432-021-03670-y}

In the original article, the Fig. 2 is published incorrectly. The correct Fig. 2 is given below.

The original article can be found online at https://doi.org/10.1007/ s00432-021-03670-y.

Udo Jeschke

udo.jeschke@med.uni-muenchen.de;

Udo.Jeschke@uk-augsburg.de

Wanting Shao

schaowt111@126.com

Christina Kuhn

Christina.kuhn@uk-augsburg.de

Doris Mayr

doris.mayr@med.uni-muenchen.de

Nina Ditsch

nina.ditsch@uk-augsburg.de

Magdalena Kailuwait

magdalena.kailuweit@swmbrk.de

Verena Wolf

verena-wolf@gmx.net

Nadia Harbeck

nadia.harbeck@med.uni-muenchen.de

Sven Mahner

sven.mahner@med.uni-muenchen.de

Vincent Cavaillès

vincent.cavailles@inserm.fr
Sophie Sixou

sophie.sixou@inserm.fr

1 Department of Obstetrics and Gynecology, Breast Center, LMU University Hospital, Munich, Germany

2 Department of Breast Center, School of Medicine, The First Affiliated Hospital, Zhejiang University, Hangzhou 310003, China

3 Department of Gynecology and Obstetrics, University Hospital Augsburg, Stenglinstr. 2, 86156 Augsburg, Germany

4 Department of Pathology, LMU Munich, Munich, Germany

5 IRCM-Institut de Recherche en Cancérologie de Montpellier, INSERM U1194, Université Montpellier, Parc Euromédecine, 208 rue des Apothicaires, F-34298 Montpellier, France

6 Faculté Des Sciences Pharmaceutiques, Université Toulouse III - Paul Sabatier, 31062 Toulouse, France

7 Cholesterol Metabolism and Therapeutic Innovations, Cancer Research Center of Toulouse (CRCT), UMR 1037, INSERM, UPS, Université de Toulouse, CNRS, 31037 Toulouse, France 
A

Total

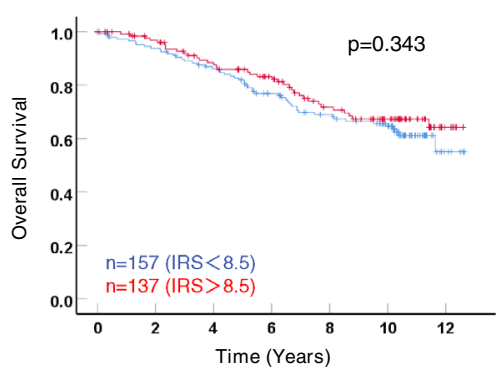

B

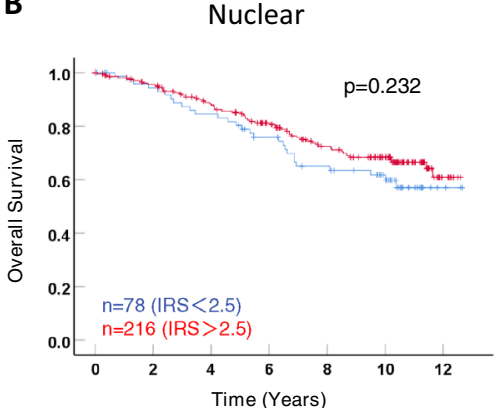

C

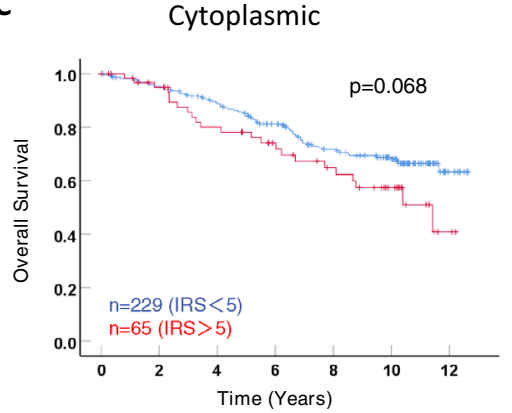

Fig. 2 Kaplan-Meier analyses of patient overall survival (OS) in the whole cohort according to nuclear, cytoplasmic, and total LXR expression. OS Kaplan-Meier curves are presented according to total (A), nuclear (B), and cytoplasmic (C) LXR expression. The optimal
IRS cut-off values for nuclear, cytoplasmic, and total LXR expression were determined as $2.5,5$, and 8.5 , respectively. The number of cases for each group is indicated in each penal

The original article has been corrected.

Publisher's Note Springer Nature remains neutral with regard to jurisdictional claims in published maps and institutional affiliations. 